Appl. Set-Valued Anal. Optim. 3 (2021), No. 2, pp. 215-220

Available online at http://asvao.biemdas.com

https://doi.org/10.23952/asvao.3.2021.2.06

\title{
A NEW LINEAR CONVERGENCE METHOD FOR A LIPSCHITZ PSEUDOMONOTONE VARIATIONAL INEQUALITY
}

\author{
R. N. NWOKOYE ${ }^{1}$, C.C. $\mathrm{OKEKE}^{2}$, Y. SHEHU ${ }^{3, *}$ \\ ${ }^{1}$ Department of Mathematics, University of Nigeria, Nsukka, Nigeria \\ ${ }^{2}$ Department of Mathematics and Applied Mathematics, \\ Sefako Makgatho Health Sciences University, Pretoria, South Africa \\ ${ }^{3}$ Department of Mathematics, Zhejiang Normal University, Jinhua, China
}

\begin{abstract}
In this paper, a new method for solving variational inequalities for which the underline cost function is strongly-pseudomonotone and Lipschitz continuous is proposed and linear convergence is obtained in real Hilbert spaces under some mild assumptions.
\end{abstract}

Keywords. Linear Convergence; Pseudomonotone operator; Variational inequality.

\section{INTRODUCTION}

Throughout this paper, $H$ is assumed to be a real Hilbert space with scalar product $\langle.,$.$\rangle and$ induced norm $\|\cdot\|$. Let $C$ be a nonempty, closed and convex subset of $H$, and let $A: H \rightarrow H$ be a continuous mapping. We consider the following variational inequality problem (for short, $\mathrm{VI}(A, C))$, which is consists of finding $x \in C$ such that

$$
\langle A x, y-x\rangle \geq 0, \quad \forall y \in C \text {. }
$$

We denote by $S$ the set of solutions of $\operatorname{VI}(A, C)(1.1)$.

It was shown in [1] that the sequence $\left\{x_{n}\right\}$ generated by the projected gradient method

$$
x_{n+1}=P_{C}\left(x_{n}-\lambda_{n} A x_{n}\right)
$$

converges linearly to the unique solution of $\mathrm{VI}(A, C)(1.1)$ provided that $A$ is a strongly pseudomonotone Lipschitz continuous mapping, and $0<a \leq \lambda_{n} \leq b<\frac{2 \eta}{L^{2}}$. The strong convergence of $\left\{x_{n}\right\}$ (but not linear convergence) was also obtained provided that $\sum_{n=1}^{\infty} \lambda_{n}=\infty$ and $\lim _{n \rightarrow \infty} \lambda_{n}=0$. It is generally known that the projected gradient method (1.2) fails when $A$ is a pseudomonotone operator.

${ }^{*}$ Corresponding author.

E-mail addresses: regina.nwokoye@unn.edu.ng (R.N. Nwokoye), chibueze.okeke87@yahoo.com (C.C. Oke ke), yekini.shehu@unn.edu.ng (Y. Shehu)

Received April 28, 2020; Accepted July 30, 2020.

(C)2021 Applied Set-Valued Analysis and Optimization 
Motivated by the extragradient method introduced by Korpelevich [2]), Khanh [3] proposed the following modified extragradient method for solving $\operatorname{VI}(A, C)(1.1)$ :

$$
\left\{\begin{array}{l}
x_{1} \in C, \\
y_{n}=P_{C}\left(x_{n}-\lambda_{n} A x_{n}\right) \\
x_{n+1}=P_{C}\left(x_{n}-\lambda_{n} A y_{n}\right), n \geq 1,
\end{array}\right.
$$

where $A$ is strongly pseudomonotone Lipschitz continuous and $0<a \leq \lambda_{n} \leq b<\frac{2 \eta}{L^{2}}$, and obtained the linear convergence of the algorithm. The strong convergence (but not linear convergence) of the sequence $\left\{x_{n}\right\}$ generated by (1.3) was obtained in [4] provided that $\sum_{n=1}^{\infty} \lambda_{n}=\infty$ and $\lim _{n \rightarrow \infty} \lambda_{n}=0$. One of the drawbacks of (1.1) is that if $P_{C}$ does not have a closed form formula, then a minimization problem has to be solved twice per iteration in implementing extragradient method (1.3). In this case, the extragradient method (1.3) for solving $\operatorname{VI}(A, C)(1.1)$ is not efficient.

In [5], Thong and Hieu studied the combination of subgradient extragradient method of Censor et al. [6] and the inertial extrapolation step: $x_{1} \in H$,

$$
\left\{\begin{array}{l}
w_{n}=x_{n}+\theta_{n}\left(x_{n}-x_{n-1}\right), \\
y_{n}=P_{C}\left(w_{n}-\lambda_{n} A w_{n}\right), \\
T_{n}:=\left\{w \in H:\left\langle w_{n}-\lambda_{n} A w_{n}-y_{n}, w-y_{n}\right\rangle \leq 0\right\} \\
x_{n+1}=P_{T_{n}}\left(w_{n}-\lambda_{n} A y_{n}\right),
\end{array}\right.
$$

and obtained strong convergence results (but no linear convergence) of $\operatorname{VI}(A, C)(1.1)$ provided that $A$ is strongly pseudomonotone and Lipschitz continuous, $\sum_{n=1}^{\infty} \lambda_{n}=\infty, \lim _{n \rightarrow \infty} \lambda_{n}=0$ and $0 \leq \theta_{n} \leq \frac{1}{10}$. This result can be reduced to the result in [7] if $\theta_{n}=0$.

The following simple example in $\mathbb{R}$ shows that both extragradient method (1.3) and inertial subgradient extragradient method (1.4) cannot converge linearly to the unique solution of $\operatorname{VI}(A, C)(1.1)$ when $\sum_{n=1}^{\infty} \lambda_{n}=\infty$ and $\lim _{n \rightarrow \infty} \lambda_{n}=0$.

Example 1.1. Take $C=\mathbb{R}$ and $A x:=x, x \in \mathbb{R}$. Then $A$ is 1 -strongly pseudomonotone and 1Lipschitz continuous. Also, $\operatorname{VI}(A, C)(1.1)$ has a unique solution $x^{*}=0$. Choose $\theta_{n}=0$ in (1.4). For any $x_{1} \neq 0$, extragradient method (1.3) and inertial subgradient extragradient method (1.4) reduce to

$$
\left\{\begin{array}{l}
y_{n}=x_{n}-\lambda_{n} x_{n} \\
x_{n+1}=x_{n}-\lambda_{n} y_{n}
\end{array}\right.
$$

that is

$$
x_{n+1}=\left(1-\lambda_{n}\right) x_{n}+\lambda_{n}^{2} x_{n}
$$

Now, with $\lim _{n \rightarrow \infty} \lambda_{n}=0$ and $x_{1} \neq 0$, one can see that $\left\{x_{n}\right\}$ does not converge linearly to $x^{*}=0$.

Motivated by the results of Thong and Hieu [5], our aim in this paper is to introduce a new projection method for $\operatorname{VI}(A, C)(1.1)$ when $A$ is strongly pseudomonotone and Lipschitz continuous, and obtain linear convergence results.

\section{PRELIMINARIES}

Definition 2.1. Recall that a mapping $A: H \rightarrow H$ is said to be

(a) $\eta$-strongly monotone on $H$ if there exists a constant $\eta>0$ such that $\langle A x-A y, x-y\rangle \geq$ $\eta\|x-y\|^{2}$ for all $x, y \in H$ 
(b) monotone on $H$ if $\langle A x-A y, x-y\rangle \geq 0$ for all $x, y \in H$;

(c) $\delta$-strongly pseudo-monotone on $H$ if there exists $\delta>0$ such that $\langle A y, x-y\rangle \geq 0 \Rightarrow$ $\langle A x, x-y\rangle \geq \delta\|x-y\|^{2}, x, y \in H$

(d) pseudomonotone on $H$ if, for all $x, y \in H,\langle A x, y-x\rangle \geq 0 \Rightarrow\langle A y, y-x\rangle \geq 0$;

(e) L-Lipschitz continuous on $H$ if there exists a constant $L>0$ such that $\|A x-A y\| \leq$ $L\|x-y\|$ for all $x, y \in H$.

Remark 2.1. Note that (a) implies (b); (a) implies (c); (c) implies (d); and (b) implies (d) in the above definitions. If $A$ is $\eta$-strongly pseudomonotone and Lipschitz continuous, it has been shown (see, e.g., [8]) that $\operatorname{VI}(A, C)(1.1)$ has unique solution.

Definition 2.2. $P_{C}$ is called the metric projection of $H$ onto $C$ if, for any point $u \in H$, there exists a unique point $P_{C} u \in C$ such that

$$
\left\|u-P_{C} u\right\| \leq\|u-y\|, \forall y \in C .
$$

$P_{C}$ satisfies (see, e.g., [9])

$$
\left\langle x-y, P_{C} x-P_{C} y\right\rangle \geq\left\|P_{C} x-P_{C} y\right\|^{2}, \forall x, y \in H .
$$

Furthermore, $P_{C} x$ is characterized by the properties

$$
P_{C} x \in C \quad \text { and } \quad\left\langle x-P_{C} x, P_{C} x-y\right\rangle \geq 0, \forall y \in C .
$$

This characterization implies that

$$
\|x-y\|^{2} \geq\left\|x-P_{C} x\right\|^{2}+\left\|y-P_{C} x\right\|^{2}, \forall x \in H, \forall y \in C .
$$

Lemma 2.1. The following statements hold in $H$ :

(i) $\|x+y\|^{2}=\|x\|^{2}+2\langle x, y\rangle+\|y\|^{2}$ for all $x, y \in H$;

(ii) $\|x+y\|^{2} \leq\|x\|^{2}+2\langle y, x+y\rangle$ for all $x, y \in H$

(iii) $\|\alpha x+\beta y\|^{2}=\alpha(\alpha+\beta)\|x\|^{2}+\beta(\alpha+\beta)\|y\|^{2}-\alpha \beta\|x-y\|^{2}, \forall x, y \in H, \alpha, \beta \in \mathbb{R}$.

Definition 2.3. Suppose that a sequence $\left\{x_{n}\right\}$ in $H$ converges in norm to $x^{*} \in H$. We say that $\left\{x_{n}\right\}$ converges to $x^{*} R$-linearly if $\limsup _{n \rightarrow \infty}\left\|x_{n}-x^{*}\right\|^{\frac{1}{n}}<1$. We say that $\left\{x_{n}\right\}$ converges to $x^{*} Q$-linearly if there exists $\mu \in(0,1)$ such that $\left\|x_{n+1}-x^{*}\right\| \leq \mu\left\|x_{n}-x^{*}\right\|$ for all sufficiently large $n$. It is well known that the $Q$-linear convergence implies the $R$-linear convergence, but the reverse implication is not true.

\section{MAIN RESUlTS}

We now give a linear convergence result via the following new method.

Theorem 3.1. Assume that A is $\eta$-strongly pseudomonotone and L-Lipschitz continuous on $H$. Let $\gamma \in\left(0, \frac{1}{2}\right), 0<\lambda<\frac{2 \eta}{L^{2}}$ and $0<\delta<\lambda\left(2 \eta-\lambda L^{2}\right)$. Suppose that $\left\{x_{n}\right\}$ is generated by: $x_{0}, x_{1} \in H$,

$$
\left\{\begin{array}{l}
y_{n}=x_{n}+\delta\left(x_{n}-x_{n-1}\right) \\
x_{n+1}=(1-\gamma) x_{n}+\gamma P_{C}\left(y_{n}-\lambda A y_{n}\right)
\end{array}\right.
$$

Then $\left\{x_{n}\right\}$ converges at least $R$-linear to the unique solution $x^{*}$ of $\operatorname{VI}(A, C)$ 
Proof. Define

$$
w_{n}=P_{C}\left(y_{n}-\lambda A y_{n}\right), \quad \forall n \geq 1 .
$$

Then

$$
\left\langle y_{n}-\lambda A y_{n}-w_{n}, u-w_{n}\right\rangle \leq 0, \quad \forall u \in C .
$$

In particular, we get

$$
\left\langle y_{n}-\lambda A y_{n}-w_{n}, x^{*}-w_{n}\right\rangle \leq 0 .
$$

Therefore,

$$
2\left\langle y_{n}-w_{n}, x^{*}-w_{n}\right\rangle \leq 2 \lambda\left\langle A y_{n}, x^{*}-w_{n}\right\rangle
$$

In view of $x^{*} \in S$, we get

$$
\left\langle A x^{*}, y-x^{*}\right\rangle \geq 0, \quad \forall y \in C .
$$

By the strong pseudomonotonicity of $A$, one gets

$$
\left\langle A y, y-x^{*}\right\rangle \geq \eta\left\|y-x^{*}\right\|^{2}, \quad \forall y \in C .
$$

Using Cauchy-Schwartz inequality, the Lipschitz continuity of $A$ and the inequality $2 a b \leq a^{2}+$ $b^{2} \forall a, b \in \mathbb{R}$, we obtain

$$
\begin{aligned}
2 \lambda\left\langle A y_{n}, x^{*}-w_{n}\right\rangle & =-2 \lambda\left\langle A w_{n}, w_{n}-x^{*}\right\rangle+2 \lambda\left\langle A y_{n}-A w_{n}, x^{*}-w_{n}\right\rangle \\
& \leq-2 \lambda \eta\left\|w_{n}-x^{*}\right\|^{2}+2 \lambda\left\|A y_{n}-A w_{n}\right\|\left\|w_{n}-x^{*}\right\| \\
& \leq-2 \lambda \eta\left\|w_{n}-x^{*}\right\|^{2}+2 \lambda L\left\|y_{n}-w_{n}\right\|\left\|w_{n}-x^{*}\right\| \\
& \leq-2 \lambda \eta\left\|w_{n}-x^{*}\right\|^{2}+\left\|y_{n}-w_{n}\right\|^{2}+(\lambda L)^{2}\left\|w_{n}-x^{*}\right\|^{2}
\end{aligned}
$$

Observe also that

$$
\begin{aligned}
2\left\langle y_{n}-w_{n}, x^{*}-w_{n}\right\rangle & =\left\|y_{n}-w_{n}\right\|^{2}+\left\|x^{*}-w_{n}\right\|^{2}-\left\|\left(y_{n}-w_{n}\right)-\left(x^{*}-w_{n}\right)\right\|^{2} \\
& =\left\|y_{n}-w_{n}\right\|^{2}+\left\|x_{n+1}-x^{*}\right\|^{2}-\left\|w_{n}-x^{*}\right\|^{2} .
\end{aligned}
$$

Substituting (3.3) and (3.4) into (3.2) gives

$$
\begin{aligned}
& \left\|y_{n}-w_{n}\right\|^{2}+\left\|w_{n}-x^{*}\right\|^{2}-\left\|y_{n}-x^{*}\right\|^{2} \\
& \leq-2 \lambda \eta\left\|w_{n}-x^{*}\right\|^{2}+\left\|y_{n}-w_{n}\right\|^{2}+(\lambda L)^{2}\left\|w_{n}-x^{*}\right\|^{2} .
\end{aligned}
$$

Therefore, $\forall n \geq 1$, we get

$$
\left(1+\lambda\left(2 \eta-\lambda L^{2}\right)\right)\left\|w_{n}-x^{*}\right\|^{2} \leq\left\|y_{n}-x^{*}\right\|^{2}
$$

i.e.,

$$
\left\|w_{n}-x^{*}\right\|^{2} \leq \frac{1}{1+\lambda\left(2 \eta-\lambda L^{2}\right)}\left\|y_{n}-x^{*}\right\|^{2} .
$$

Observe that

$$
\begin{aligned}
\left\|y_{n}-x^{*}\right\|^{2} & =\left\|(1+\delta)\left(x_{n}-x^{*}\right)-\delta\left(x_{n-1}-x^{*}\right)\right\|^{2} \\
& =(1+\delta)\left\|x_{n}-x^{*}\right\|^{2}-\delta\left\|x_{n-1}-x^{*}\right\|^{2}+\delta(1+\delta)\left\|x_{n}-x_{n-1}\right\|^{2}
\end{aligned}
$$


and

$$
\begin{aligned}
\left\|x_{n+1}-x^{*}\right\|^{2} & =\left\|(1-\gamma)\left(x_{n}-x^{*}\right)+\gamma\left(w_{n}-x^{*}\right)\right\|^{2} \\
& =(1-\gamma)\left\|w_{n}-x^{*}\right\|^{2}+\gamma\left\|w_{n}-x^{*}\right\|^{2}-\gamma(1-\gamma)\left\|x_{n}-w_{n}\right\|^{2} \\
& =(1-\gamma)\left\|x_{n}-x^{*}\right\|^{2}+\gamma\left\|w_{n}-x^{*}\right\|^{2}-\frac{(1-\gamma)}{\gamma}\left\|x_{n+1}-x_{n}\right\|^{2}
\end{aligned}
$$

which implies from (3.5) that

$$
\begin{aligned}
\left\|x_{n+1}-x^{*}\right\|^{2} \leq & (1-\gamma)\left\|x_{n}-x^{*}\right\|^{2}+\frac{\gamma}{1+\lambda\left(2 \eta-\lambda L^{2}\right)}\left\|y_{n}-x^{*}\right\|^{2} \\
& -\frac{(1-\gamma)}{\gamma}\left\|x_{n+1}-x_{n}\right\|^{2} .
\end{aligned}
$$

Using (3.6) and (3.8), we have

$$
\begin{aligned}
& \left\|x_{n+1}-x^{*}\right\|^{2} \\
\leq & (1-\gamma)\left\|x_{n}-x^{*}\right\|^{2}+\frac{\gamma}{1+\lambda\left(2 \eta-\lambda L^{2}\right)}\left((1+\delta)\left\|x_{n}-x^{*}\right\|^{2}-\delta\left\|x_{n-1}-x^{*}\right\|^{2}\right. \\
& \left.+\delta(1+\delta)\left\|x_{n}-x_{n-1}\right\|^{2}\right)-\frac{(1-\gamma)}{\gamma}\left\|x_{n+1}-x_{n}\right\|^{2} \\
= & (1-\gamma)\left\|x_{n}-x^{*}\right\|^{2}+\frac{\gamma(1+\delta)}{1+\lambda\left(2 \eta-\lambda L^{2}\right)}\left\|x_{n}-x^{*}\right\|^{2} \\
& -\frac{\delta \gamma}{1+\lambda\left(2 \eta-\lambda L^{2}\right)}\left\|x_{n-1}-x^{*}\right\|^{2}+\frac{\gamma \delta(1+\delta)}{1+\lambda\left(2 \eta-\lambda L^{2}\right)}\left\|x_{n}-x_{n-1}\right\|^{2} \\
& -\frac{(1-\gamma)}{\gamma}\left\|x_{n+1}-x_{n}\right\|^{2} .
\end{aligned}
$$

Re-arranging gives

$$
\begin{aligned}
\left\|x_{n+1}-x^{*}\right\|^{2}+\frac{(1-\gamma)}{\gamma}\left\|x_{n+1}-x_{n}\right\|^{2} \leq & \left(1-\gamma+\frac{\gamma(1+\delta)}{1+\lambda\left(2 \eta-\lambda L^{2}\right)}\right)\left\|x_{n}-x^{*}\right\|^{2} \\
& +\frac{\gamma \delta(1+\delta)}{1+\lambda\left(2 \eta-\lambda L^{2}\right)}\left\|x_{n}-x_{n-1}\right\|^{2} .
\end{aligned}
$$

Put

$$
\xi^{2}=\frac{1}{1+\lambda\left(2 \eta-\lambda L^{2}\right)} \in(0,1) .
$$

Then (3.9) becomes

$$
\begin{aligned}
\left\|x_{n+1}-x^{*}\right\|^{2}+\left\|x_{n+1}-x_{n}\right\|^{2} \leq & \left\|x_{n+1}-x^{*}\right\|^{2}+\frac{(1-\gamma)}{\gamma}\left\|x_{n+1}-x_{n}\right\|^{2} \\
\leq & \left(1-\gamma\left(1-\xi^{2}(1+\delta)\right)\right)\left\|x_{n}-x^{*}\right\|^{2} \\
& +\gamma \xi^{2} \delta(1+\delta)\left\|x_{n}-x_{n-1}\right\|^{2} \\
\leq & \left(1-\gamma\left(1-\xi^{2}(1+\delta)\right)\right) \\
& \times\left(\left\|x_{n}-x^{*}\right\|^{2}+\left\|x_{n}-x_{n-1}\right\|^{2}\right),
\end{aligned}
$$


due to $\gamma \in\left(0, \frac{1}{2}\right)$ and

$$
\frac{\gamma \xi^{2} \delta(1+\delta)}{1-\gamma\left(1-\xi^{2}(1+\delta)\right)}<1
$$

From $0<\delta<\lambda\left(2 \eta-\lambda L^{2}\right)$ and $\gamma \in\left(0, \frac{1}{2}\right)$, we have $0<\gamma\left(1-\xi^{2}(1+\delta)\right)<1$. Denote

$$
a_{n}:=\left\|x_{n}-x^{*}\right\|^{2}+\left\|x_{n}-x_{n-1}\right\|^{2} \text {. }
$$

Then (3.10) becomes

$$
a_{n+1} \leq\left(1-\gamma\left(1-\xi^{2}(1+\delta)\right)\right)^{n} a_{1}
$$

which implies that

$$
\left\|x_{n+1}-x^{*}\right\|^{2} \leq a_{n+1} \leq\left(1-\gamma\left(1-\xi^{2}(1+\delta)\right)\right)^{n} a_{1} .
$$

Hence the proof.

Corollary 3.1. Assume that $A$ is $\eta$-strongly monotone and L-Lipschitz continuous on H. Let $\gamma \in\left(0, \frac{1}{2}\right), 0<\lambda<\frac{2 \eta}{L^{2}}$ and $0<\delta<\lambda\left(2 \eta-\lambda L^{2}\right)$. Suppose that $\left\{x_{n}\right\}$ is generated by (3.1). Then $\left\{x_{n}\right\}$ converges at least $R$-linear to the unique solution $x^{*}$ of $V I(A, C)$.

Remark 3.1. Our proposed method (3.1) seems simpler than the methods (1.3) and (1.4), and the linear convergence is obtained. Our method does not require extra projection onto the feasible set $C$ as in (1.3) and no extra projection onto the half-space as in (1.4).

\section{Acknowledgments}

The authors are grateful to the referees for the useful suggestions which improved the presentation of this paper.

\section{REFERENCES}

[1] P. D. Khanh, P. T. Vuong, Modified projection method for strongly pseudomonotone variational inequalities, J. Global Optim. 58 (2014), 341-350.

[2] G.M. Korpelevich, The extragradient method for finding saddle points and other problems, Ekonomika i Mat. Metody 12 (1976), 747-756.

[3] P. D. Khanh, Convergence rate of a modified extragradient method for pseudomonotone variational inequalities, Vietnam J. Math. 45 (2017), 397-408.

[4] P.D. Khanh, A new extragradient method for strongly pseudomonotone variational inequalities, Numer. Funct. Anal. Optim. 37 (2016), 1131-1143.

[5] D. V. Thong, D. V. Hieu, Inertial extragradient algorithms for strongly pseudomonotone variational inequalities, J. Comput. Appl. Math. 341 (2018), 80-98.

[6] Y. Censor, A. Gibali, S. Reich, The subgradient extragradient method for solving variational inequalities in Hilbert space, J. Optim. Theory Appl. 148 (2011), 318-335.

[7] D. V. Hieu, D. V. Thong, New extragradient-like algorithms for strongly pseudomonotone variational inequalities, J. Glob. Optim. 70 (2018), 385-399.

[8] J. C. Yao, Variational inequalities with generalized monotone operators, Math. Oper. Res. 19 (1994), $691-705$.

[9] H.H. Bauschke, P.L. Combettes, Convex Analysis and Monotone Operator Theory in Hilbert Spaces, CMS Books in Mathematics, Springer, New York, 2011. 